\title{
Total Synthesis of Geranylgeranylglyceryl Phosphate Enantiomers: Substrates for Characterization of 2,3-0- Digeranylgeranylglyceryl Phosphate Synthase
}

\author{
Honglu Zhang $^{a}$, Kyohei Shibuya $^{\mathrm{b}}$, Hisashi Hemmi $^{\mathrm{b}}$, Tokuzo Nishino ${ }^{\mathrm{b}}$, and Glenn D. \\ Prestwich ${ }^{\mathrm{a}}{ }^{*}$ \\ 1 Department of Medicinal Chemistry, The University of Utah, 419 Wakara Way, Suite 205, Salt Lake City, \\ Utah 84108-1257, USA \\ b Department of Biomolecular Engineering, Tohoku University, Aoba-yama 07, Sendai, miyagi 980-8579, \\ Japan
}

\begin{abstract}
In order to determine the enantioselectivity of $(S)$-2,3-di-O-geranylgeranylglyceryl phosphate synthase (DGGGPS) from the thermoacidophilic archaeon Sulfolobus solfataricus, we developed an efficient enantioselective route to the enantiomeric geranylgeranylglyceryl phosphates $(R)$-GGGP and $(S)$-GGGP. Previous routes to these substrates involved enzymatic conversions, due to the lability of the polyprenyl chains towards common phosphorylation reaction conditions. The synthesis described herein employs a mild trimethyl phosphite/carbon tetrabromide oxidative phosphorylation to circumvent this problem. In contrast to previous results suggesting that only $(S)$-GGGP can act as the prenyl-acceptor substrate, both $(R)$-GGGP and $(S)$-GGGP were found to be substrates for DGGGPS.
\end{abstract}

All living organisms have been classified into three primary kingdoms: archaebacteria, eubacteria, and eukaryotes. ${ }^{1}$ While the membrane lipids in eubacteria and eukaryotes are composed of glyceryl esters of fatty acids, archaebacterial membrane lipids contain isopranyl glyceryl ethers. 2,3 The core structures of archaeal membrane lipids, including diether and bipolar tetraether lipids, contain fully-reduced $\mathrm{C}_{20}$ or $\mathrm{C}_{25}$ prenyl groups. ${ }^{4,5}$ Biosynthesis of the isoprene moieties in the core lipids follows the mevalonate pathway used also by eubacteria and eukaryotes. ${ }^{6-8}$ The prenyl transfer reactions responsible for building the isoprenoid diphosphates, e.g., geranylgeranyl diphosphate (GGPP), are catalyzed by a family of prenyltransferases. ${ }^{9-12}$ As shown in Figure 1, the biogenesis of the core-structure of the archaeal membrane lipids starts with the prenyl transfer reaction catalyzed by $(S)-3-O$ geranylgeranylglyceryl phosphate [(S)-GGGP] synthase, which selectively uses $(S)$-glyceryl phosphate as the prenyl acceptor (Fig. 1). Then, the product is utilized as the presumed acceptor substrate for the biosynthesis of $(S)$-2,3-Di- $O$-geranylgeranylglyceryl phosphate (DGGGP), an advanced intermediate of archaeal membrane lipids. ${ }^{13}$

To date, only an enzyme-assisted synthesis of (S)-GGGP has been reported. ${ }^{11}$ An enantiospecific chemical synthesis of both individual enantiomers of GGGP was required to validate this biosynthetic hypothesis, due in part to the acid-sensitive geranylgeranyl group of GGGP. In order to fully characterize the substrate selectivity of DGGGP synthase (DGGGPS), we developed a mild and effective route to the two GGGP enantiomers. The instability of 
GGGP indeed posed significant challenges, and the biological results with the enantiomers were unexpected.

The synthesis of $(S)$-GGGP $(\mathbf{8})$ is summarized in Scheme 1. Treatment of the $(2 E, 6 E, 10 E)$ geranylgeraniol 1 with $\mathrm{Ph}_{3} \mathrm{P} / \mathrm{CBr}_{4}$ at $25^{\circ} \mathrm{C}$ afforded geranylgeranyl bromide $2 .{ }^{14} \mathrm{Next}$, $(S)$ solketal was alkylated with geranylgeranyl bromide by using $\mathrm{KH}$ as base, to give ether $\mathbf{3}$ in $73 \%$ yield. ${ }^{11}$ The reported HCl/THF method to remove the acetonide ${ }^{13}$ resulted in a complex mixture containing the desired product in low yield. The desired diol $\mathbf{4}$ was thus prepared in $75 \%$ yield using $p$-TsOH in methanol. ${ }^{15,16}$ In order to obtain selective phosphorylation of the primary hydroxyl, we first tried a strategy involving protection of the secondary hydroxyl as a silyl ether. Thus, both hydroxyl groups of diol $\mathbf{4}$ were protected as TBS ethers (TBSCl, imidazole, anhydrous DMF). ${ }^{15,16}$ Subsequently, the more labile primary TBS ether was selectively removed at $\mathrm{rt}$ by using HF.Py (HF.Py/Py/THF=1:2:5). Unfortunately, phosphorylation of the primary alcohol under standard conditions (dimethylphosphoryl chloride, $t$ - $\left.\mathrm{BuOK}, \mathrm{CH}_{2} \mathrm{Cl}_{2}\right)^{15,16}$ failed to give the desired product due to the lability of the polyene system.

A second strategy proved more successful. The use of the trimethyl phosphite/carbon tetrabromide oxidative phosphorylation method 17 was deemed sufficiently mild to permit phosphorylation without damage to the geranylgeranyl moiety. Treatment of diol $\mathbf{4}$ with 1.1 eq of $\mathrm{CBr}_{4}$ and 1.2 eq of $\mathrm{P}(\mathrm{OMe})_{3}$ gave selective phosphorylation of the primary alcohol to give the protected phosphate 7 . Essentially no bisphosphate product was detected.

The next challenge in this synthesis was liberation of the free phosphate monoester from the protected triester. We first tried TMSBr, a standard deprotecting reagent for removal of methyl and ethyl groups in the synthesis of acyl migration-prone lysophosphatidic acid derivatives. 15,16 However, GGGP did not survive this strong Lewis acid. By using a solution of TMSBr in 2,4,6-trimethylpyridine (sym-collidine), ${ }^{18-20}$ we obtained the desired monophosphate in the acidic form. Titration with $1 \mathrm{~N}$ aq $\mathrm{NaOH}$ afforded $(S)$-GGGP $(\mathbf{8})$ as the stabilized sodium salt.

In order to determine the enantioselectivity of DGGGPS, both the enantiomers $(S)$-GGGP $(\mathbf{8})$ and $(R)$-GGGP $(\mathbf{1 2})$, were required. Starting with $(R)$-solketal, $(R)$-GGGP $(\mathbf{1 2})$ was synthesized using the successful route as summarized in Scheme 2.

DGGGPS is a member of the UbiA prenyltransferase family that can catalyze the transfer of a prenyl group to its biological acceptor substrate $(S)$-GGGP $(\mathbf{8})$. With the enantiomeric substrates $(R)$-GGGP $(\mathbf{1 2})$ and $(S)$-GGGP $(\mathbf{8})$ in hand, we determined the activity of DGGGPS toward each of these substrates. From the results of radio-HPLC analysis (Fig. 2) and reversedphase TLC analysis (Fig. 3), we found the DGGGP and presumably its enantiomer were formed in the reactions using $(S)$-GGGP $(\mathbf{8})$ and $(R)$-GGGP $(\mathbf{1 2})$, respectively. In these reactions, the starting reagent $\left[{ }^{14} \mathrm{C}\right]$-GGPP was formed first from $\left[{ }^{14} \mathrm{C}\right]$ isopentenyl diphosphate and $(E, E)$ farnesyl diphosphate by the activity of GGPS. Then, $\left[{ }^{14} \mathrm{C}\right]$-GGPP was used as the prenyl donor substrate for DGGGPS. Thus, the results demonstrated that the $\mathrm{C}_{20}$-prenyl group of GGPP could be transferred to either of the two GGGP enantiomers by the action of DGGGPS. (S)GGGP seems to be marginally preferred in Fig. 2, while the $(R)$-enantiomer appeared to be preferred in Fig. 3. These results are reproducible but qualitative; the GGGPs produced are quite labile. Nevertheless, to our surprise, both $(R)$-GGGP $(\mathbf{1 2})$ and $(S)$-GGGP $(\mathbf{8})$ were accepted at a comparable extent as substrates for DGGGPS. During the biosynthesis of archaeal membrane lipids, GGGPS catalyzes the transfer of prenyl groups from GGPP to $(S)$-glyceryl phosphate in formation of $(S)$-GGGP $(\mathbf{8})$, and both the ether linkage between $(S)$-GGGP $(\mathbf{8})$ and another geranylgeranyl group are formed under the control of DGGGPS. GGGPSs are known to have strict substrate preferences: $(R)$-glyceryl phosphate is a very poor substrate. 
13, 21 Thus, our results strongly suggest that the chirality of archaeal membrane lipid is determined by GGGPS, not by DGGGPS. However, $(R)$-GGGP $(\mathbf{1 2})$ and $(S)$-GGGP $(\mathbf{8})$ will be important tools for more detailed analysis of the specific activity and enantioselectivity of DGGGPS in future studies.

In conclusion, $(S)$-GGGP and $(R)$-GGGP were each synthesized by a five-step procedure starting from the $(2 E, 6 E, 10 E)$-geranylgeraniol and the appropriate enantiomer of solketal. A regioselective phosphorylation of diol 4 was achieved using $\mathrm{CBr}_{4} / \mathrm{P}(\mathrm{OMe})_{3}$, and the instability problem of geranylgeranyl group was circumvented by judicious selection of mild reaction conditions. The LKC18 reversed phase-TLC analysis and radio-HPLC analysis have shown that the DGGGPS can catalyze the transfer of prenyl group to the secondary hydroxy groups of both $(R)$-GGGP and $(S)$-GGGP.

\section{Supplementary Material}

Refer to Web version on PubMed Central for supplementary material.

\section{Acknowledgements}

We thank Dr. Y. Xu (U Utah) and G. Jiang (U Utah) for many helpful discussions. We thank the NIH (NS29632 to GDP) and grants-in-aid from the Ministry of Education, Culture, Sports, Science, and Technology of Japan for financial support of this work.

\section{References}

1. Woese CR. Sci Am 1981;244:98-122.

2. Langworthy TA, Tornabene TG, Holzer G. Zentralbl Bakteriol Mikrobiol Hyg, Abt I, Orig C 1982;3:228-244.

3. Langworthy TA, Pond JL. Syst Appl Microbiol 1986;7:253-257.

4. Kates M. Prog Chem Fats Other Lipids 1978;15:301-342. [PubMed: 358256]

5. Heathcock CH, Finkelstein BL, Aoki T, Poulter CD. Science 1985;229:862-864. [PubMed: 3927485]

6. De Rosa M, De Rosa S, Gambacorta A. Phytochemistry 1977;16:1909-1912.

7. De Rosa M, Gambacorta A, Nicolaus B. Phytochemistry 1980;19:791-793.

8. Moldoveanu N, Kates M. Biochim Biophys Acto 1988;960:164-182.

9. Poulter, CD. Biochemistry of Cell Walls and Membranes in Fungi. Kuhn, PJ.; Trinci, APJ.; Jung, MJ.; Goosey, MW.; Copping, LG., editors. Springer-Verlag; Berlin, Heidelberg: 1990. p. 169-188.

10. Poulter, CD. Biosynthesis of Isopreniod compounds. Rilling, HC.; Porter, JW.; Spurgeon, SL., editors. I. Wiley; New York: 1981. p. 161-224.

11. Zhang D, Poulter CD. J Am Chem Soc 1993;115:1270-1277.

12. Ohnuma, S-i; Suzuki, M.; Nishino, T. J Biol Chem 1994;269:14792-14797. [PubMed: 8182085]

13. Hemmi H, Shibuya K, Takahashi Y, Nakayama T, Nishino T. J Biol Chem 2004;279:50197-50203. [PubMed: 15356000]

14. Tokumasu MAH, Hiraga Y, Kojima S, Ohkta K. J Chem Soc Perkin Trans 1 1999:489-496.

15. Xu Y, Prestwich GD. J Org Chem 2002;67:7158-7161. [PubMed: 12354017]

16. Xu Y, Qian L, Prestwich GD. J Org Chem 2003;68:5320-5330. [PubMed: 12816494]

17. Oza VB, Corcoran RC. J Org Chem 1995;60:3680-3684.

18. Cermak DM, Wiemer DF, Lewis K, Hohl RJ. Bioorg Med Chem 2000;8:2729-2737. [PubMed: 11131164]

19. Macchia M, Jannitti N, Gervasi G, Danesi R. J Med Chem 1996;39:1352-1356. [PubMed: 8691464]

20. Magnin DR, Biller SA, Dickson JK Jr, Logan JV, Lawrence RM, Chen Y, Sulsky RB, Ciosek CP Jr, Harrity TW, Jolibois KG, et al. J Med Chem 1995;38:2596-2605. [PubMed: 7629799]

21. Zhang D, Poulter CD. J Org Chem 1993;58:3919-3922. 


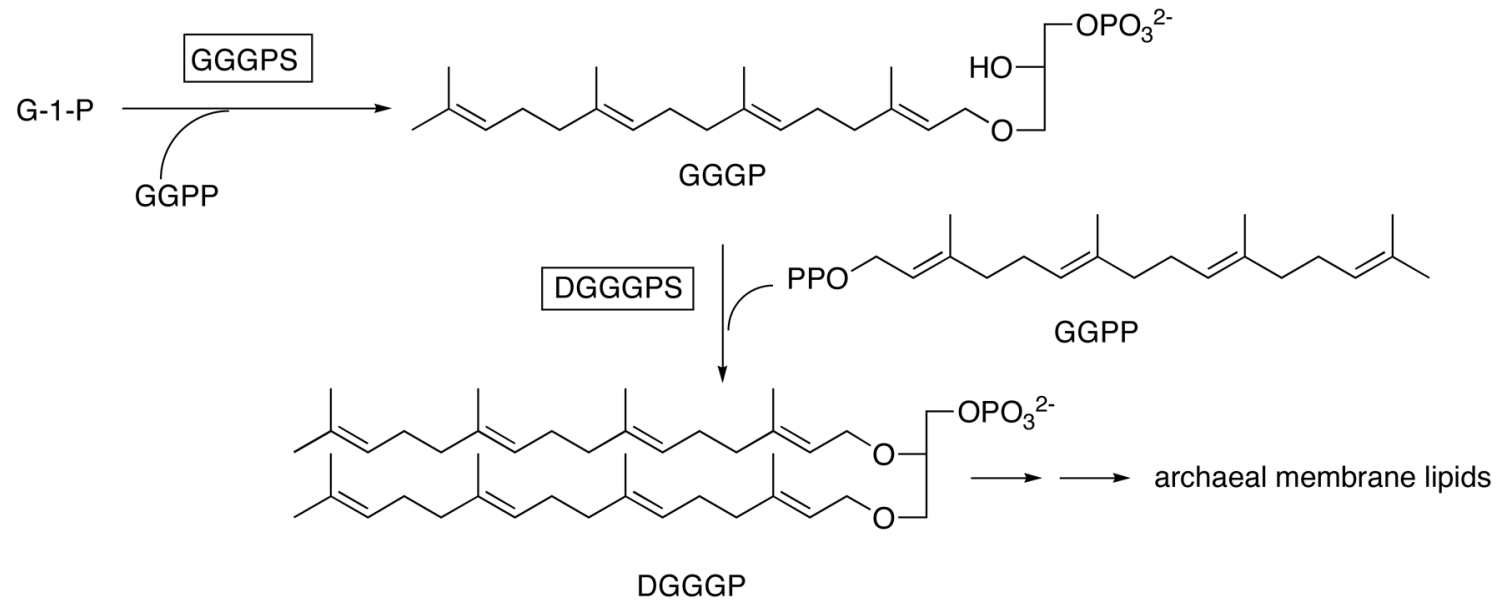

Figure 1.

Biosynthesis of DGGGP, a key intermediate for archaeal membrane lipids. Key:G-1-P, $(S)$ glyceryl phosphate; GGGPS, (S)-GGGP synthase; DGGGPS, DGGGP synthase 

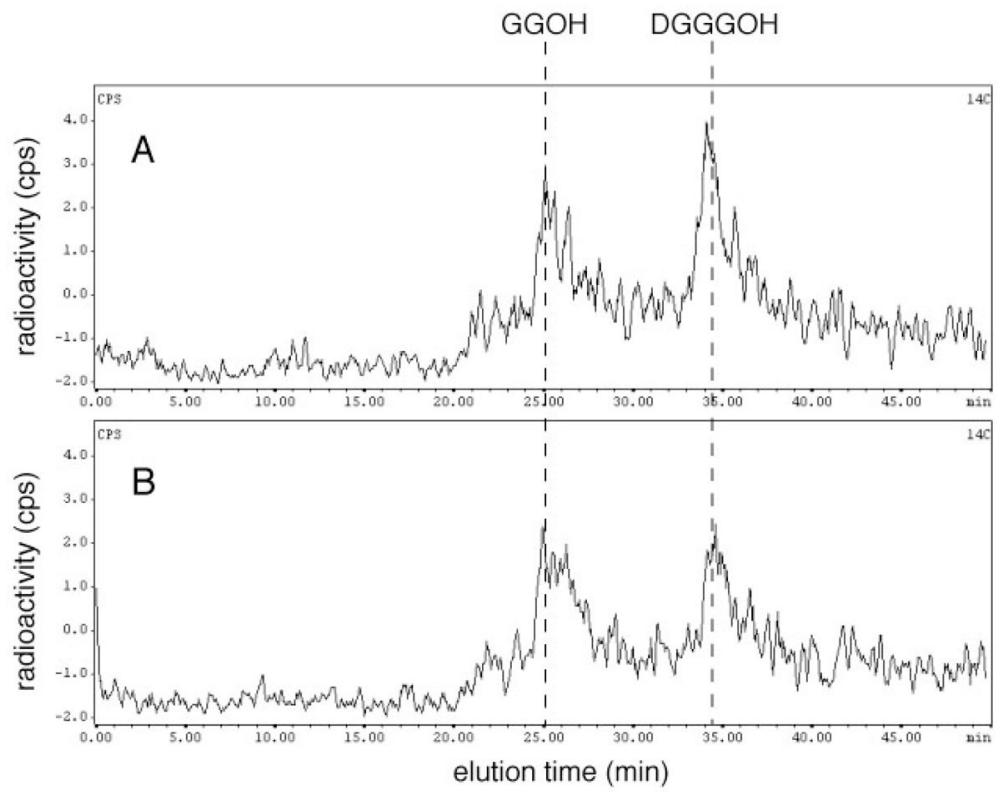

Figure 2.

Radio-HPLC analysis of 1-butanol-extracts from the enzyme reaction with $\left[{ }^{14} \mathrm{C}\right]-\mathrm{GGPP}$ synthase and DGGGPS, using $0.5 \mathrm{nmol}\left[{ }^{14} \mathrm{C}\right]$-isopentenyl diphosphate, $0.5 \mathrm{nmol}(E, E)$ farnesyl diphosphate, $0.4 \mu \mathrm{mol}(S)$-GGGP (A) or $(R)$-GGGP (B) as substrates. GGOH, geranylgeraniol; DGGGOH, digeranylgeranylglycerol. 

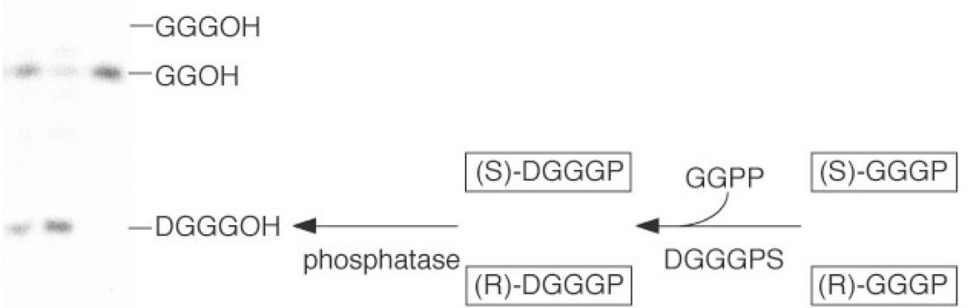

123

Figure 3.

Autoradiogram of TLC, from left to right: 1. (S)-GGGP; 2. (R)-GGGP; 3. without an acceptor substrate. GGGOH, geranylgeranylglycerol; GGOH, geranylgeraniol; DGGGOH, digeranylgeranylglycerol; Ori, origin; S.f., solvent front. 
<smiles>CCC(=O)c1ccccc1</smiles>

1<smiles>CCCC(C)=CCOCC(O)CO</smiles><smiles>[3H]/C(=C\COC[C@H](O)COP(=O)(OC)OC)CC/C=C(\C)CC/C=C(\C)CCC=C(C)C</smiles>

4

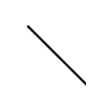<smiles>CC(C)=CCC/C(C)=C/CC/C(C)=C/CC/C(C)=C/CBr</smiles>

2

(S)-soketal, $\mathrm{KH}$ THF, $0^{\circ} \mathrm{C}$ to rt $73 \%$

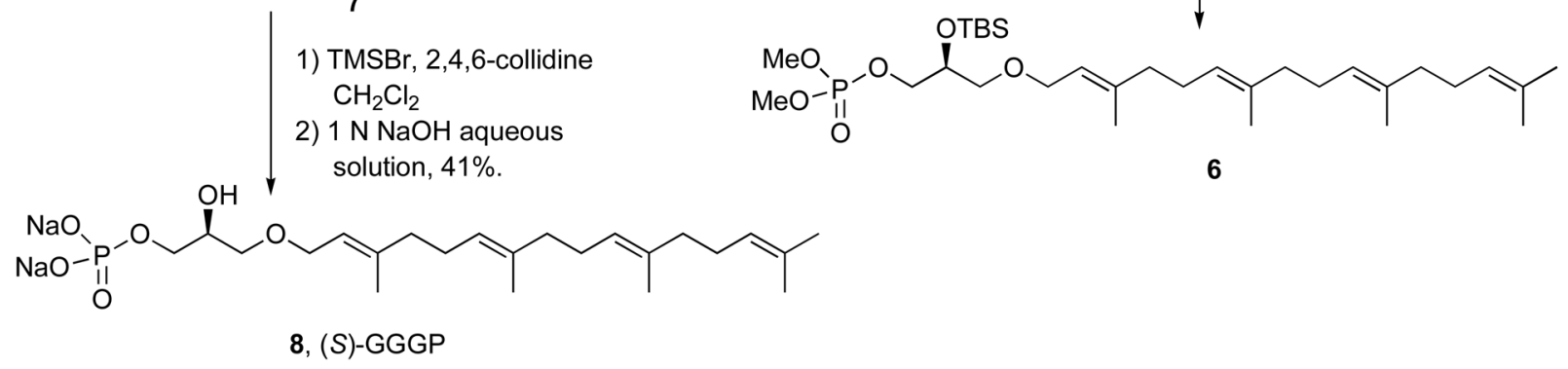

Scheme 1.

Synthesis of (S)-GGGP (8). 

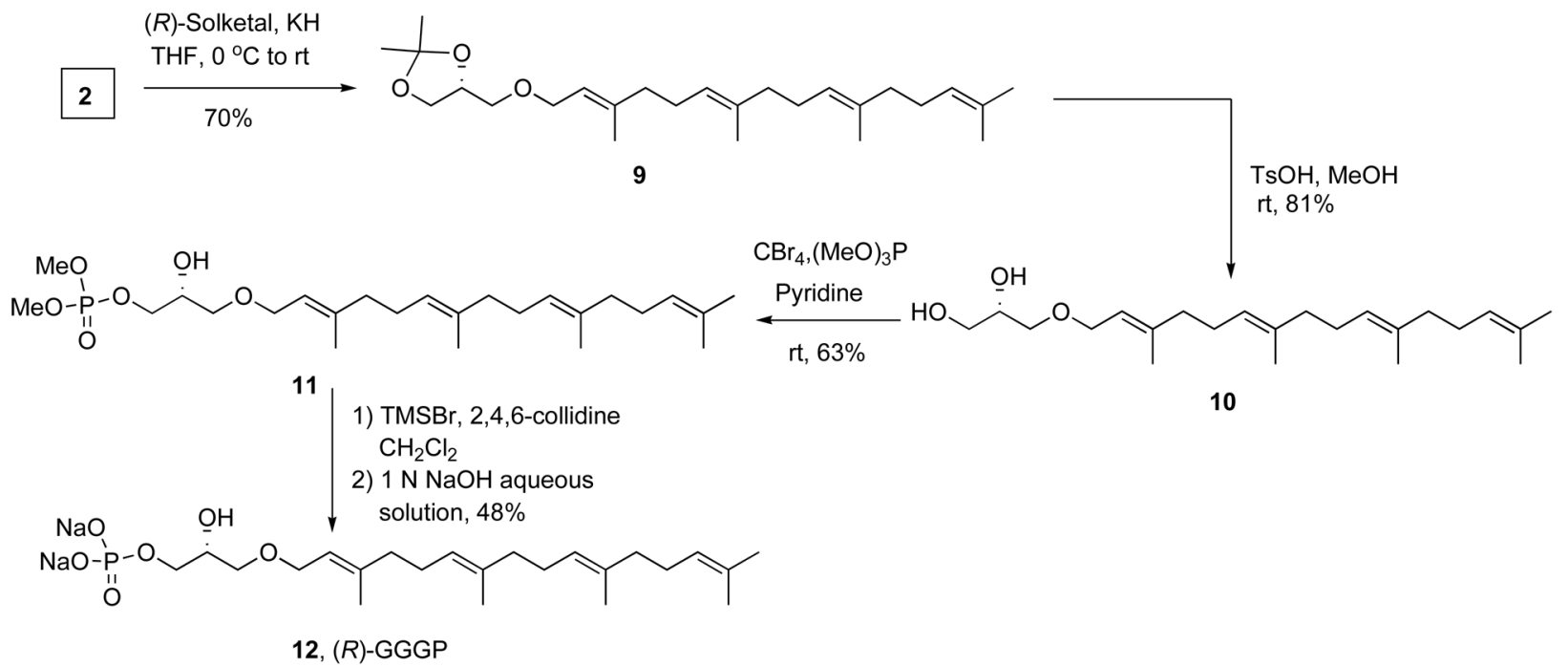

10

Scheme 2.

Synthesis of $(R)$-GGGP (12). 